\title{
Drop impact of shear thickening liquids
}

\author{
François Boyer, Enrique Sandoval-Nava, Jacco H. Snoeijer, J. Frits Dijksman, and Detlef Lohse* \\ Physics of Fluids Group, MESA+ Institute, and Faculty of Science and Technology, University of Twente, \\ P.O. Box 217, 7500 AE Enschede, Netherlands
}

(Received 12 January 2016; published 2 May 2016)

\begin{abstract}
The impact of drops of concentrated non-Brownian suspensions (cornstarch and polystyrene spheres) onto a solid surface is investigated experimentally. The spreading dynamics and maximal deformation of the droplet of such shear thickening liquids are found to be markedly different from the impact of Newtonian drops. A particularly striking observation is that the maximal deformation is independent of the drop velocity and that the deformation suddenly stops during the impact phase. Both observations are due to the shear thickening rheology of the suspensions, as is explained theoretically from a balance between the kinetic energy and the viscously dissipated energy, from which we establish a scaling relation between the maximal deformation of the drop and rheological parameters of concentrated suspensions.
\end{abstract}

DOI: 10.1103/PhysRevFluids.1.013901

The impact of liquid drops on solid surfaces is a classical experiment in interfacial hydrodynamics [1] and has received increasing attention from a broad community (including applied mathematics, soft matter physics, and chemical engineering) [2,3]. Impact is relevant for a large number of industrial processes (e.g., inkjet printing [4], spray coating, and pesticide delivery [5]) and touches upon the fundamental challenges of spreading, splashing, and bouncing that arise in this paradigmatic experiment. Recent studies have been dedicated to providing a better understanding of the spreading dynamics, in particular on superhydrophobic [6], superheated [7], and/or microstructured surfaces [8,9], and have used a variety of experimental techniques (Fourier-transform profilometry [10], particle image velocimetry [11], and high-speed color interferometry [12]) to get new insights into the complex hydrodynamics of an impacting droplet. Splashing remains the least understood regime as it has been revealed to depend on a large number of parameters [13-15] and involves an intricate coupling between air and liquid flows [16-18].

Despite the complex mechanical response of a large class of fluids used in relevant applications (paint, blood for forensic studies, printing of organic light-emitting diodes, and colloids for the increasingly popular three-dimensional inkjet printing [19]), most drop impact studies have focused on Newtonian liquids. However, as we will show in this paper, non-Newtonian properties affect the drop impact dynamics dramatically. Conversely, such impact studies may be applied to accurately extract the complex rheology of such materials. It has already been shown that viscoelastic properties have a strong influence on the retraction dynamics after impact [20]. More recently, yield stress and shear thinning fluids have been extensively studied and successful scaling laws have been introduced to relate the rheological properties to the maximal deformation of the drop [21,22].

In this paper we investigate drop impact for concentrated suspensions, which are known to exhibit shear thickening effects. We reveal that during the impact phase, the liquid freezes into a strongly deformed state (Fig. 1). Remarkably, this maximum deformation turns out to be completely independent of the impact velocity. We systematically study the parametric dependence of maximal deformation with the drop size and particle concentration and derive scaling arguments based on the balance between kinetic energy and viscously dissipated energy. Rheological measurements are used to characterize the shear thickening regime and provide scaling laws that are in excellent

*d.lohse@utwente.nl 
agreement with all observations, such as the independence of the spreading on the impact velocity.

The experimental setup has been previously described in [22]. A drop of suspension is slowly generated out of a capillary needle driven by a syringe pump until it detaches and falls under its own weight. The released drop has a diameter close to the capillary length and quickly relaxes to a spherical shape. Changing the needle's outer diameter allows one to control the falling drop diameter $D_{0}$ from 1.8 to $3.5 \mathrm{~mm}$. The drop impact velocity $U_{0}$ is varied by releasing the drop from different heights, in the range of $0.3-3.0 \mathrm{~m} / \mathrm{s}$. The impacted surface is a clean and smooth microscope glass slide. The impact dynamics is visualized from the side with a high-speed camera (Photron Fastcam APX-RS) at 20000 frames/s with a macrolens. The intense and homogeneous backlighting needed is obtained with a $42-\mathrm{W}$ xenon light source and a light diffuser.

Two types of suspensions are used to perform the experiments: (i) commercially available cornstarch (Duryea, typical diameter $\bar{d}=5-20 \mu \mathrm{m}$ and density $\rho_{p}=1.683 \mathrm{~g} / \mathrm{cm}^{3}$ ) and (ii) polystyrene spherical (PS) particles (Microbeads, diameter $d=20 \mu \mathrm{m}$ and density $\rho=1.051 \mathrm{~g} / \mathrm{cm}^{3}$ ). Both are dispersed in density-matched aqueous solutions of cesium chloride (dynamic viscosity $\eta_{f}=1 \mathrm{mPa} \mathrm{s}$ ) $[23,24]$. The suspensions are prepared by slowly pouring solid particles into the carrier fluid and vigorously stirring the mixture with a spatula. The samples are finally sonicated for a few minutes before use in the experiments for even better mixing. Even at longer time scales (hours), no settling or creaming of the particle phase is observed. Control of the volume fraction $\phi_{0}$ (defined as the ratio of the solid particle volume to the total suspension volume) is achieved by weighing both particles and fluid. Cornstarch suspensions are prepared at different volume fractions: $33 \%, 35 \%$, and $38 \%$. Note that these concentrations are still well below those necessary for walking on cornstarch [25]. In addition, a suspension of PS particles was prepared at 55\%. For both cornstarch and PS particles, the large size of the suspended particles ensures that Brownian motion and colloidal interactions can be safely neglected and such systems are thus called granular suspensions. The use of particles of two chemically different materials and sphericity allows one to test the universality of the drop impact behavior.

Figure 1 shows snapshots of a typical drop impact experiment (35\% cornstarch suspension, $D_{0}=2.80 \mathrm{~mm}$, and $U_{0}=1.04 \mathrm{~m} \mathrm{~s}^{-1}$ ) and illustrates the peculiar behavior of granular suspensions. In sharp contrast to low-viscosity fluids $[10,11,27]$, the spreading phase of the impacting drop abruptly stops shortly after impact. The drop is then frozen in a deformed state and no macroscopic deformation of the drop interface is observed at intermediate time scales. While this is qualitatively similar to very-high-viscosity Newtonian drops, the impacting suspension is seen to exhibit a remarkable feature: The droplet shapes become irregular, with a breaking of the azimuthal symmetry. At longer times $(t>40 \mathrm{~ms}$ in the present experiment), the drop relaxes to a spherical cap shape: This slow relaxation dynamics (not shown here) depends on the surface properties of the suspension and the substrate, but does not depend on the impact parameters $\left(U_{0}, D_{0}\right)$ and will not be documented in this paper. In the following we will focus on the short-time-scale impact dynamics, i.e., at times comparable to the characteristic impact time scale $t_{0}=D_{0} / U_{0}$. The image sequence shown in Fig. 1 also emphasizes a characteristic feature of the drop impact dynamics of suspensions: Whereas the so-called spreading ratio is seen to reach a maximum value $D_{\text {max }} / D_{0}$ ranging typically from 2 to 10 for Newtonian fluids, the diameter of the base area here remains on the order of $D_{0}$, i.e., $D_{\max } / D_{0} \sim 1$. Since the spreading of suspension drops exhibits a symmetry breaking, $D_{\max }$ depends on variations of the wetted area at the instant of contact; the drop height $h$ is chosen as a more suitable quantity to characterize drop deformation upon impact.

From image analysis, the height $h$ of the drop (at the apex) is determined for each frame of the high-speed movie and a characteristic time evolution is shown in Fig. 1, in dimensionless units $h / D_{0}$ vs $U_{0} t / D_{0}$. It can be described as a two-stage process: (i) a first kinematic stage, where $h$ is seen to decrease linearly with time at a constant speed $U_{0}$ (black dashed line, $h / D_{0}=1-U_{0} t / D_{0}$ ), as if the motion of the drop apex is not altered by the impact event, and (ii) a frozen state, where $h$ stays constant at a plateau value $\varepsilon_{m} D_{0}$ for times longer than $D_{0} / U_{0}$. The crossover time $\tau$ is 
(a)

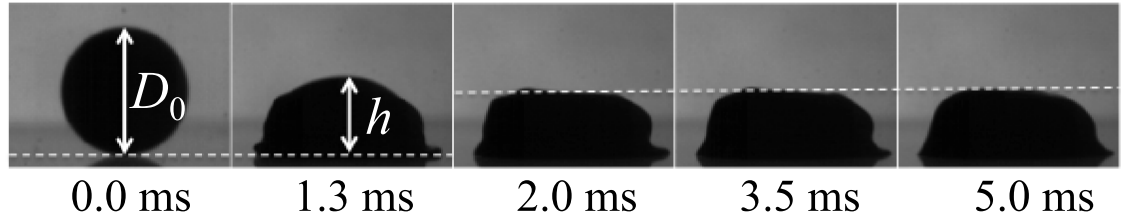

(b)

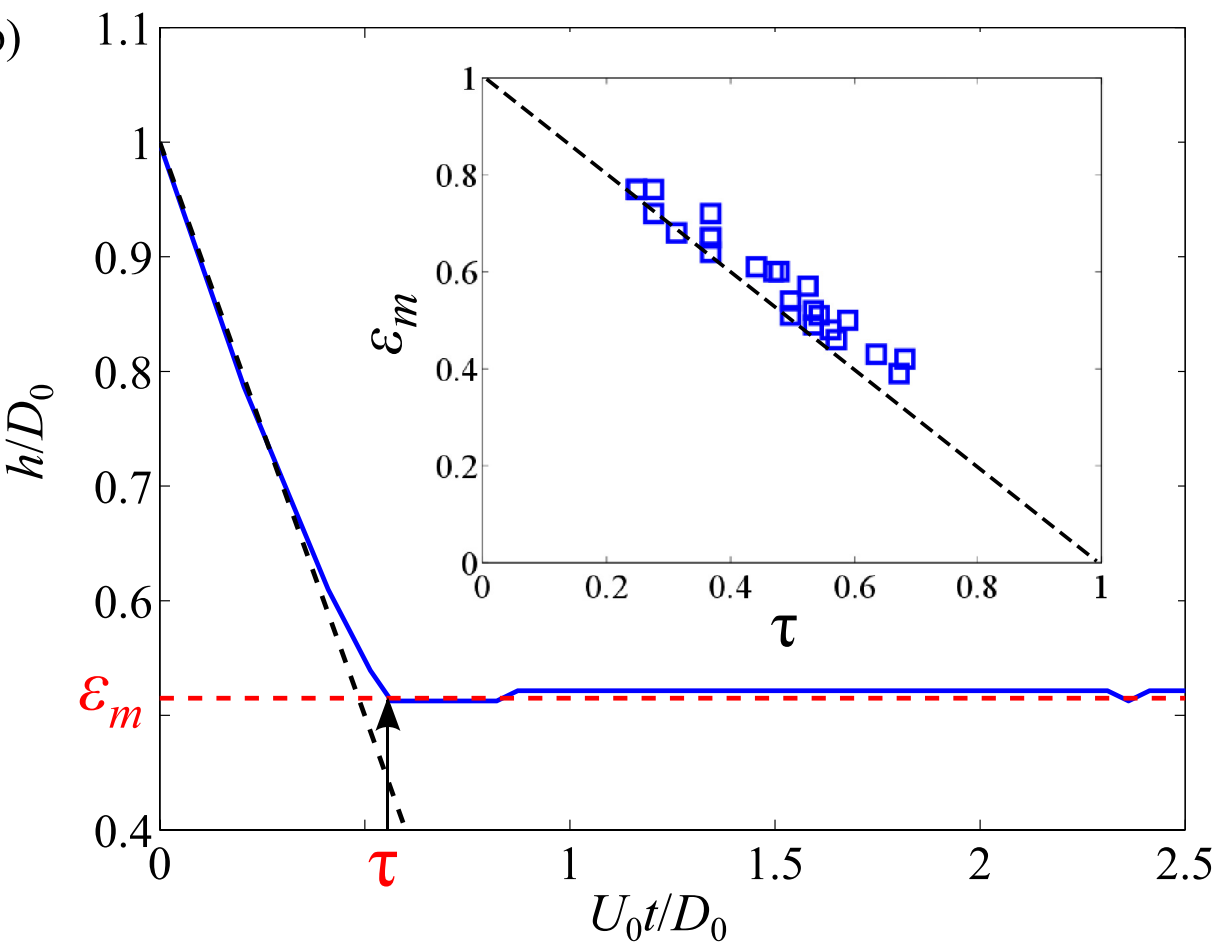

(c)

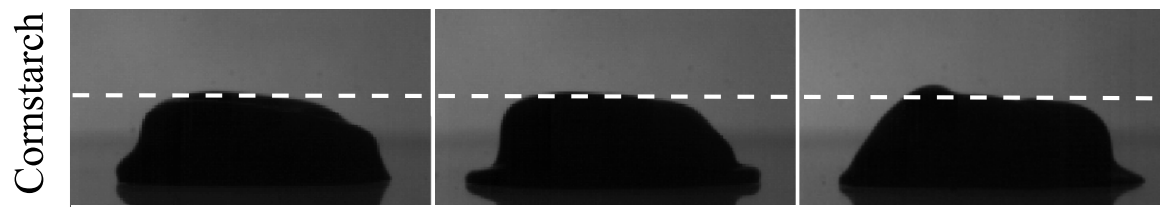

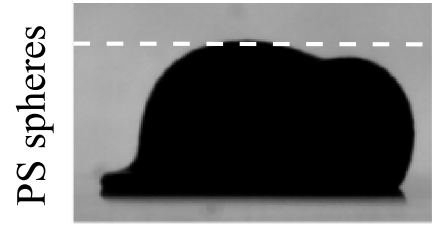

$0.37 \mathrm{~m} / \mathrm{s}$

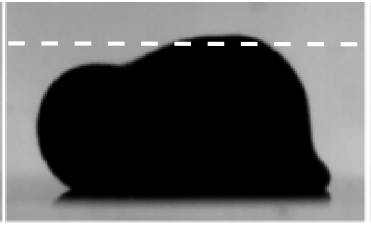

$1.02 \mathrm{~m} / \mathrm{s}$

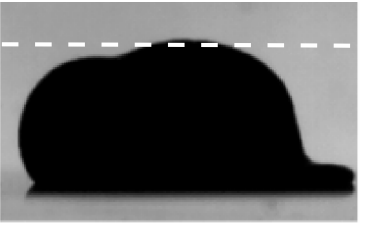

$2.91 \mathrm{~m} / \mathrm{s}$

FIG. 1. (a) Image sequence of a drop of cornstarch suspension impacting a glass plate $\left(D_{0}=2.80 \mathrm{~mm}\right.$, $U_{0}=1.04 \mathrm{~m} / \mathrm{s}$, and $\phi_{0}=35 \%$ ). Part of the images consists of reflection of the drops on the glass surface. (b) Dimensionless drop height $h / D_{0}$ as a function of dimensionless time $U_{0} t / D$. The experiment can be divided into a kinematic stage (dashed black line) and the frozen stage (red dashed line) defining the plateau value $\epsilon_{m}$, which is shown in the inset as a function of the dimensionless crossover time $\tau$, also defined in the main plot. (c) Snapshots of frozen drops of cornstarch $\left(D_{0}=2.80 \mathrm{~mm}\right.$ and $\left.\phi_{0}=35 \%\right)$ and PS particle $\left(D_{0}=2.23 \mathrm{~mm}\right.$ and $\phi_{0}=55 \%$ ) suspensions, for different impact velocities. At later times, the drops will slowly spread and recover an axisymmetric shape. See Ref. [26]. 


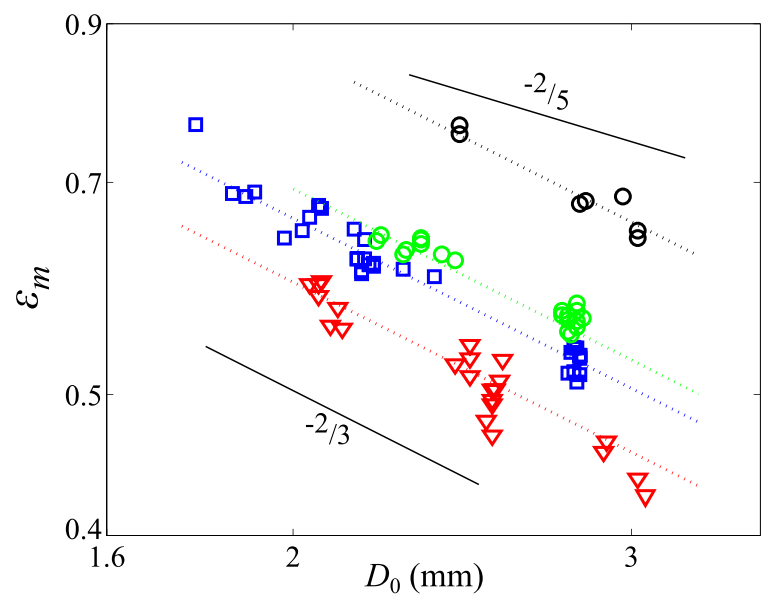

FIG. 2. Dimensionless height $\varepsilon_{m}$ as a function of drop diameter $D_{0}$ (logarithmic scale), for different cornstarch volume fractions 33\% (red triangles), 35\% (blue squares), and 38\% (black circles) and for a 55\% PS particle suspension (green circles).

defined as the first moment where $h$ reaches the plateau value. An ideally sharp transition between the two stages would then lead to $\varepsilon_{m}=1-\tau$, which is reasonably verified (within 10\%) in the inset of Fig. 1.

As emphasized, the breaking of axisymmetry is the reason that the deformation of the impinging drop is more appropriately described by the height of the drop $h$ rather than by its spreading diameter. As a result, its maximal deformation is here characterized by the plateau value $\varepsilon_{m}$ (minimal dimensionless height when the frozen state is reached). For both cornstarch and PS particle suspensions, the influence of the impact speed $U_{0}$ is studied by systematically varying the drop release height. Snapshots of frozen drops (at maximal deformation) for three impact velocities are shown in Fig. 1. Strikingly, the maximal deformation of the drop turns out to be completely independent of the impact speed over a wide range of $U_{0}$. Again, this finding is drastically different from the case of Newtonian fluids. Given the relatively large viscosity of the suspensions [at low shear rate $\eta \sim 50-100 \mathrm{mPa}$ s (see Fig. 3)], viscous forces will dominate over surface tension and the maximal deformation will be given by a balance between the drop initial kinetic energy (before impact) and viscously dissipated energy (during the spreading phase) $[6,10,11]$. Expressed in terms of $\varepsilon_{m}$, the resulting prediction for Newtonian liquids reads

$$
\varepsilon_{m} \propto \mathrm{Re}^{-2 / 5},
$$

where $\operatorname{Re}=\rho U_{0} D_{0} / \eta$ is the drop impact Reynolds number.

Clearly, the impact velocity independence observed for suspensions differs from the variation in $U_{0}^{-2 / 5}$ found for Newtonian viscous fluids. Note that velocity independence is observed for both types of particles suspensions employed, indicating a generic mechanism that is irrespective of chemical interactions and particle shape.

The influence of the other control parameters $D_{0}$ and $\phi_{0}$ is shown in Fig. 2. For a given concentration in cornstarch particles $\phi_{0}$, the dimensionless minimal height $\varepsilon_{m}$ is a decreasing function of the initial drop diameter $D_{0}$. Clearly, the experimental findings are different from the Newtonian result $D_{0}^{-2 / 5}$ and are more accurately described by a $D_{0}^{-2 / 3}$ scaling that will be derived below. As intuitively expected, more concentrated suspensions exhibit higher minimal thickness $\varepsilon_{m}$ (smaller deformations).

These observations reveal an obvious effect of the non-Newtonian properties of concentrated suspensions on the drop impact dynamics. We thus need to incorporate the actual mechanical stress 


\section{DROP IMPACT OF SHEAR THICKENING LIQUIDS}

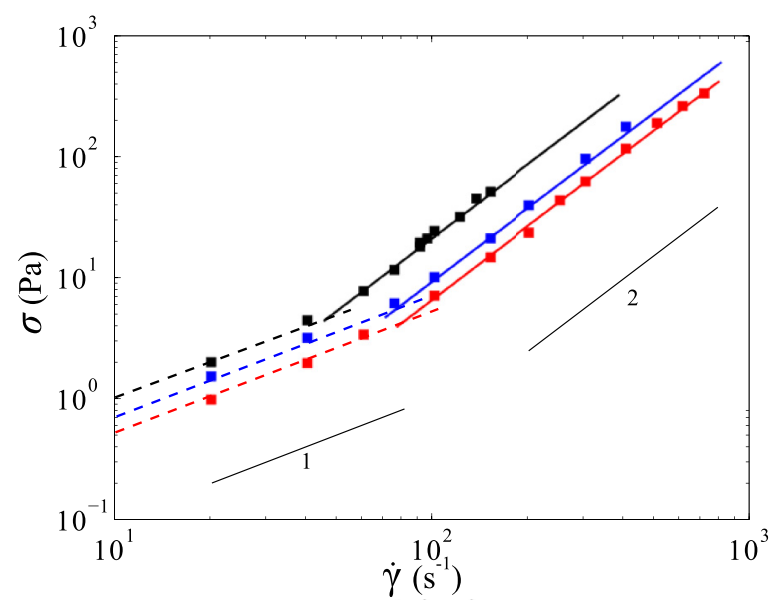

FIG. 3. Flow curves of cornstarch suspensions of different volume fractions: 33\% (red squares), $35 \%$ (blue squares), and 38\% (black squares). Dashed (solid) lines are the Newtonian (shear thickening) regimes.

response of these complex fluids and revisit the classical scaling arguments. At the concentrations studied here steady-state rheology should still be applicable [28]; at even higher concentrations we expect transient effects to be important [25]. The shear rheology of cornstarch suspensions is studied using a cylindrical Couette rheometer (Anton Paar Rheolab QC, CC39 geometry) and the resulting flow curves are shown in Fig. 3. All measurements are taken again after mixing the suspensions with a spatula to provide a homogeneous and reproducible initial state. A time evolution of the measured shear stress is observed and suggests that particle migration occurs and the suspensions quickly become inhomogeneous in local concentration (classically, particles migrate away from the inner cylinder in cylindrical geometries $[29,30])$. Therefore, to ensure that this dynamical process stays negligible (as can be expected on the very short time scale of drop impacts), the reported values in Fig. 3 are taken at early times of shear (i.e., averaged over the first ten rotations). For all cornstarch concentrations, the flow curves exhibit two regimes: (i) At low shear rates, the shear stress $\sigma$ is linear in shear rate $\dot{\gamma}$ (Newtonian behavior) with an effective viscosity depending solely on the particle volume fraction and (ii) at high shear rates, the shear stress is found to be quadratic in the shear rate. This second regime corresponds to the shear thickening regime (as the effective viscosity $\eta_{\text {eff }} \equiv \sigma / \dot{\gamma} \propto \dot{\gamma}$ is an increasing function of the shear rate), which is a well-known effect in cornstarch suspensions and more generally in particulate systems. The shear rheology of PS particle suspensions has been extensively studied [24] and has been shown to exhibit the same quadratic regime at high shear rates: As already shown in [23], the rheologies of cornstarch and spherical particle suspensions are thus found to be very similar. An estimate of the minimum shear rate $\dot{\gamma}_{0}$ experienced by an impacting drop is given by $\dot{\gamma}_{0}=U_{0} / D_{0}$, which is always larger than $10^{2} \mathrm{~s}^{-1}$ under our experimental conditions. Therefore, our drop impact experiments only probe this shear thickening regime.

The quadratic dependence observed here suggests a regime where inertia of the particles dominates over viscous forces at the particle scale. This has already been observed for concentrated non-Brownian (i.e., large particles of size $d$ ) suspensions and is known as Bagnoldian scaling, first proposed for granular media from dimensional arguments. This continuous transition to shear thickening also indicates that the volume fractions investigated here are sufficiently below the jamming point to avoid more severe shear thickening effects, for which linking any global rheology to local constitutive laws remained controversial $[23,24]$. We therefore fit the quadratic regime to the form

$$
\sigma=\kappa(\phi) \dot{\gamma}^{2}
$$




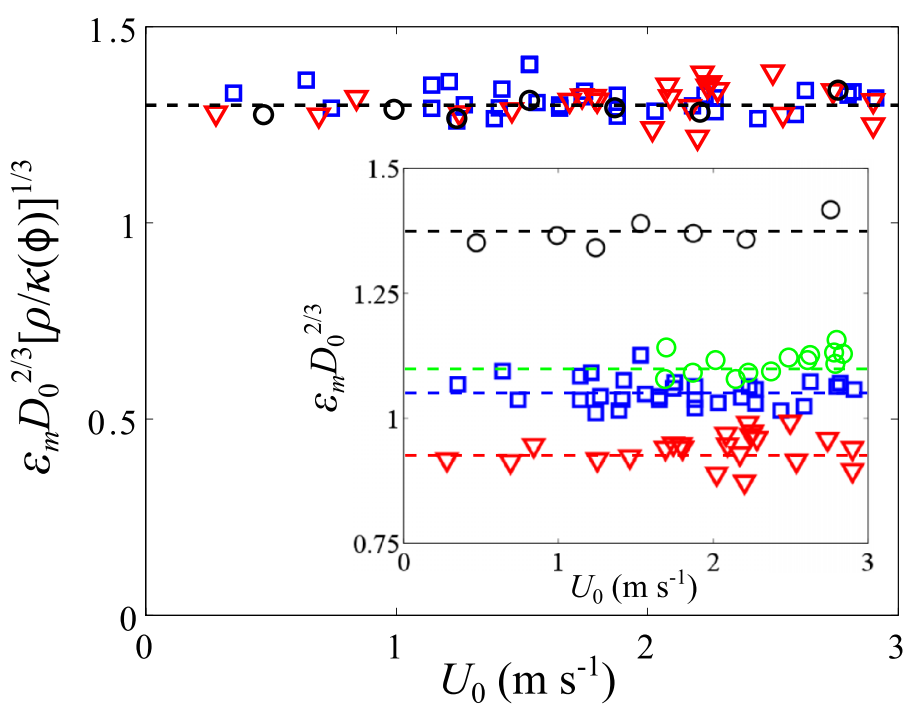

FIG. 4. Rescaled minimal height $\varepsilon_{m} D_{0}^{2 / 3}[\rho / \kappa(\phi)]^{1 / 3}$ as a function of impact velocity $U_{0}$ for different cornstarch volume fractions: $33 \%$ (red triangles), 35\% (blue squares), and 38\% (black circles). The inset shows the rescaled minimal height $\varepsilon_{m} D_{0}^{2 / 3}$, with added data for PS droplets (green circles).

and revisit the scaling argument for drop impact, taking into account this shear thickening rheology. The idea is that the initial kinetic energy of the drop is annihilated by viscous dissipation in the drop. The rate of dissipation per unit volume is computed as $\sigma \dot{\gamma}=\kappa(\phi) \dot{\gamma}^{3}$. The strain can be estimated as $\left(1-\epsilon_{m}\right) / \epsilon_{m}$, giving a strain rate $\dot{\gamma} \propto U_{0} / \epsilon_{m} D_{0}$ during the deformation phase. Since the impact time scales proportionally to $\left(1-\epsilon_{m}\right) D_{0} / U_{0}$, the total amount of dissipated energy becomes

$$
\frac{\left(1-\varepsilon_{m}\right) D_{0}}{U_{0}} \int d^{3} \mathbf{r} \sigma \dot{\gamma} \propto \kappa(\phi)\left(\frac{U_{0}}{\varepsilon_{m} D_{0}}\right)^{3}\left(\frac{\left(1-\varepsilon_{m}\right) D_{0}^{4}}{U_{0}}\right) .
$$

Equating this to the kinetic energy $\rho D_{0}^{3} U_{0}^{2}$, we obtain the maximum deformation

$$
\frac{\varepsilon_{m}}{\left(1-\varepsilon_{m}\right)^{1 / 3}} \approx \varepsilon_{m} \propto\left(\frac{\kappa(\phi)}{\rho}\right)^{1 / 3} D_{0}^{-2 / 3} .
$$

In agreement with experimental observations, the maximal deformation is found to be independent of the impact velocity $U_{0}$ in stark contrast to Newtonian fluids.

Defining a non-Newtonian Reynolds number would have also lead to the same statement: For complex fluids that follow $\sigma=K \dot{\gamma}^{n}$, the generalized Reynolds number reads $\operatorname{Re}_{n}=\rho U_{0}^{2-n} D_{0}^{n} / K$ [21]. In the present case, the shear thickening exponent is $n=2$, which cancels any dependence of the generalized Reynolds number on the velocity $U_{0}$ and then predicts the same observed behavior.

The dependence of the minimal thickness $\varepsilon_{m}$ on the drop diameter obtained in Eq. (4) is indeed found to be in very good agreement with our observations (Fig. 2). This allows us to recast the experimental results to further emphasize the independence of the spreading on the impact speed. In the inset of Fig. 4, we find that $\varepsilon_{m} D_{0}^{2 / 3}$ is indeed perfectly constant over the range of investigated impact speeds (one order of magnitude) for all suspensions.

We further test our scaling argument by using the rheology $\kappa(\phi)$ for three different volume fractions of cornstarch, as obtained from independent rheological measurement. Indeed, all 
experimental data can be scaled onto a single horizontal line (Fig. 4), suggesting a universal numerical prefactor in Eq. (4). As a result, the present drop impact experiments of cornstarch suspensions are shown to be accurately modeled by

$$
\varepsilon_{m}=1.3 \mathrm{Re}_{\mathrm{ST}}^{-1 / 3}
$$

where $\operatorname{Re}_{\mathrm{ST}}=\rho D_{0}^{2} / \kappa(\phi)$ is a generalized Reynolds number for quadratic shear thickening fluids $(n=2)$. Applying (5) to the PS data (Fig. 4, green circles), the estimated $\kappa$ is consistent with rheological data obtained by magnetic resonance imaging (MRI) [24] (see the Appendix).

As a conclusion, the drop impact of concentrated non-Brownian suspensions onto a solid surface exhibits clear differences from Newtonian fluids: Both the dynamics and final state show unusual behaviors, the most striking finding being the velocity independence of the maximal deformation. Such observation is of primary interest for numerous applications where full control of the drop size after impact is required: In this context, suspensions are an interesting class of materials for which maximal deformation depends only on initial drop size.

Revisiting classical energetic arguments while integrating the complex rheology of shear thickening suspensions leads to successful scaling laws. This gives the exciting perspective that drop impact can be used as a rheological tool: In the present case, the velocity-independent maximal deformation signals a quadratic constitutive law. Fitting the data then gives access to the rheological quantity $\kappa(\phi)$, which is usually difficult to obtain (see the Appendix). This opens the way to develop a drop impact rheometry for extreme flow conditions, of course only on the time scale probed by the droplet impact.

Note added. Recently, a related impact experiment of concentrated colloidal suspension drops on solid surfaces was published [31]. Though these experiments show some similarities to ours, there are also major differences (much higher particle volume fraction, particles that are more than three order of magnitude smaller in volume, different chemical nature of the particles, etc.) and a detailed comparison between these two sets of experiments goes beyond the scope of the present paper. Clearly, the parameter space for these sorts of experiments is considerable and must still be explored.

We thank Stef Carelsen for help with rheological measurements and Marie-Jean Thoraval for many discussions. This work was funded by the Dutch Polymer Institute under the "Inkjet-Printing of Suspensions" project.

\section{APPENDIX: RHEOLOGICAL MEASUREMENTS ON DENSE PS PARTICLE SUSPENSIONS}

Here we consider the rheological measurement on dense PS particle suspensions, as obtained by Fall et al. [24]. While standard rheology does not work for this system, Fall et al. were able to obtain proper stress-strain curves using MRI. We show that our drop impact experiment can be used to estimate the rheology and that it gives consistent results. The data from Fall et al. show for polystyrene suspensions a transition of shear stress $\sigma$ from a linear to a quadratic dependence on shear rate $\dot{\gamma}$. Similar to our own measurement on cornstarch, we fitted the coefficient of the quadratic regime $\kappa \dot{\gamma}^{2}$ for each volume fraction $\phi$. Two things must be noted here. First, the measurements [24] were done at concentrations slightly above $55 \%$. Second, the particles were $40-\mu \mathrm{m}$-diam particles, rather than our 20- $\mu$ m-diam particles. Following the theory underlying the quadratic rheology [24], the prefactor $\kappa \sim d^{2}$, where $d$ is the diameter, thus giving an extra factor of 4 when comparing (20-40)- $\mu$ m-sized particles.

Taking this into account, we can consider the green data points in the inset of Fig. 4. In order for the PS data to collapse in the main figure [using the prediction of Eq. (5)], one would require $\kappa=6.3 \times 10^{-4} \mathrm{~kg} / \mathrm{m}$. For the data from Fall et al., including the above-mentioned factor of 4 , it 


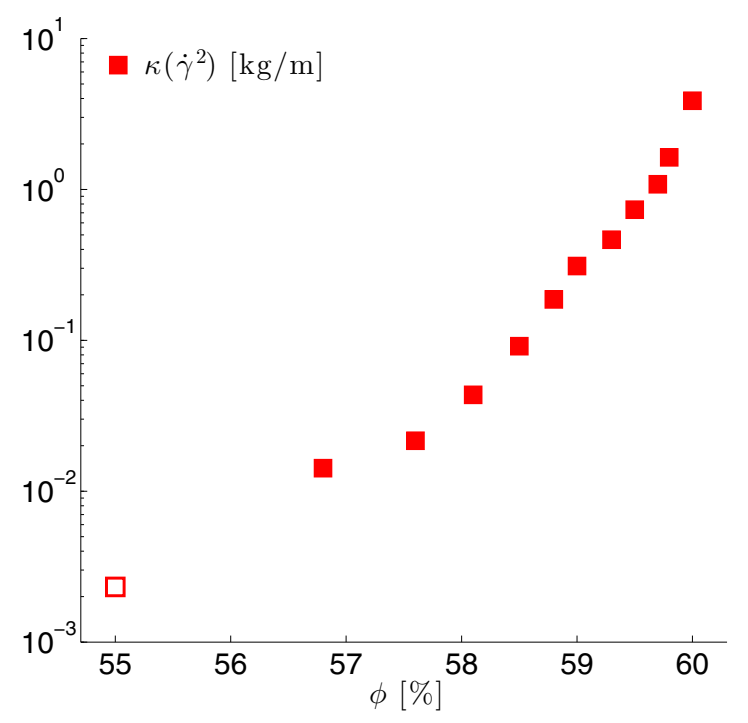

FIG. 5. Prefactor $\kappa(\phi)$ from the rheological law $\sigma=\kappa \dot{\gamma}^{2}$, estimated from the experimental data published by Fall et al. [24]. Our impact experiments were performed at $\phi=55 \%$; the corresponding value estimated from the impact experiment is reported as the open square.

would suggest $\kappa=2.5 \times 10^{-3} \mathrm{~kg} / \mathrm{m}$. The fitted $\kappa$ for different volume fractions are reported in Fig. 5 as closed squares. The open square corresponds to the value inferred from our impact experiment $\kappa=2.5 \times 10^{-3} \mathrm{~kg} / \mathrm{m}$. Indeed, the result derived from impact measurement is consistent with the data from Fall et al. [24].

[1] A. M. Worthington, On the forms assumed by drops of liquids falling vertically on a horizontal plate, Proc. R. Soc. London 25, 261 (1876); A second paper on the forms assumed by drops of liquids falling vertically on a horizontal plate, 25, 498 (1876).

[2] S. Josserand and S. T. Thoroddsen, Drop impact on solid surface, Annu. Rev. Fluid Mech. 48, 365 (2016).

[3] A. L. Yarin, Drop impact dynamics: Splashing, spreading, receding, bouncing..., Annu. Rev. Fluid Mech. 38, 159 (2006).

[4] H. Wijshoff, The dynamics of the piezo inkjet printhead operation, Phys. Rep. 491, 77 (2010).

[5] V. Bergeron, D. Bonn, J.-Y. Martin, and L. Vovelle, Controlling droplet deposition with polymer additives, Nature (London) 405, 772 (2000).

[6] C. Clanet, C. Béguin, D. Richard, and D. Quéré, Maximal deformation of an impacting drop, J. Fluid Mech. 517, 199 (2004).

[7] A.-L. Biance, C. Pirat, and C. Ybert, Drop fragmentation due to hole formation during Leidenfrost impact, Phys. Fluids 23, 022104 (2011).

[8] T. Tran, H. J. J. Staat, A. Prosperetti, C. Sun, and D. Lohse, Drop Impact on Superheated Surfaces, Phys. Rev. Lett. 108, 036101 (2012).

[9] T. Tran, H. J. J. Staat, A. Susarrey-Arce, T. C. Foertsch, A. van Houselt, H. J. G. E. Gardeniers, A. Prosperetti, D. Lohse, and C. Sun, Droplet impact on superheated micro-structured surfaces, Soft Matter 9, 3272 (2013).

[10] G. Lagubeau, M. A. Fontelosa, C. Josserand, A. Maurel, V. Pagneux, and P. Petitjeans, Spreading dynamics of drop impacts, J. Fluid Mech. 713, 50 (2012).

[11] H. Lastakowski, F. Boyer, A.-L. Biance, C. Pirat, and C. Ybert, Bridging local to global dynamics of drop impact onto solid substrates, J. Fluid Mech. 747, 103 (2014). 


\section{DROP IMPACT OF SHEAR THICKENING LIQUIDS}

[12] R. C. A. van der Veen, T. Tran, D. Lohse, and C. Sun, Direct measurements of air layer profiles under impacting droplets using high-speed color interferometry, Phys. Rev. E 85, 026315 (2012).

[13] K. Range and F. Feuillebois, Influence of surface roughness on liquid drop impact, J. Colloid Interface Sci. 203, 16 (1998).

[14] P. Tsai, R. C. A. van der Veen, M. van de Raa, and D. Lohse, How micropatterns and air pressure affect splashing on surfaces, Langmuir 26, 16090 (2010).

[15] J. C. Bird, S. S. H. Tsai, and H. A. Stone, Inclined to splash: Triggering and inhibiting a splash with tangential velocity, New J. Phys. 11, 063017 (2009).

[16] L. Xu, W. W. Zhang, and S. R. Nagel, Drop Splashing on a Dry Smooth Surface, Phys. Rev. Lett. 94, 184505 (2005).

[17] W. Bouwhuis, R. C. A. van der Veen, T. Tran, D. L. Keij, K. G. Winkels, I. R. Peters, D. van der Meer, C. Sun, J. H. Snoeijer, and D. Lohse, Maximal Air Bubble Entrainment at Liquid-Drop Impact, Phys. Rev. Lett. 109, 264501 (2012).

[18] G. Riboux and J. M. Gordillo, Experiments of Drops Impacting a Smooth Solid Surface: A Model of the Critical Impact Speed for Drop Splashing, Phys. Rev. Lett. 113, 024507 (2014); The diameters and velocities of the droplets ejected after splashing, J. Fluid Mech. 772, 630 (2015).

[19] J. A. Lewis, Direct ink writing of 3D functional materials, Adv. Funct. Mater. 16, 2193 (2006).

[20] D. Bartolo, A. Boudaoud, G. Narcy, and D. Bonn, Dynamics of Non-Newtonian Droplets, Phys. Rev. Lett. 99, 174502 (2007).

[21] L.-H. Luu and Y. Forterre, Drop impact of yield-stress fluids, J. Fluid Mech. 632, 301 (2009).

[22] M. Guémas, A. G. Marin, and D. Lohse, Drop impact experiments of non-Newtonian liquids on microstructured surfaces, Soft Matter 8, 10725 (2012).

[23] E. Brown and H. M. Jaeger, Dynamic Jamming Point for Shear Thickening Suspensions, Phys. Rev. Lett. 103, 086001 (2009).

[24] A. Fall, A. Lemaître, F. Bertrand, D. Bonn, and G. Ovarlez, Shear Thickening and Migration in Granular Suspensions, Phys. Rev. Lett. 105, 268303 (2010).

[25] S. R. Waitukaitis and H. M. Jaeger, Impact-activated solidification of dense suspensions via dynamic jamming fronts, Nature (London) 487, 205 (2012).

[26] See Supplemental Material at http://link.aps.org/supplemental/10.1103/PhysRevFluids.1.013901 for the corresponding movies (cornstarch, $\phi_{0}=35 \%: U_{0}=0.37,1.02$, and $2.91 \mathrm{~m} / \mathrm{s}$ ).

[27] J. Eggers, M. A. Fontelos, C. Josserand, and S. Zaleski, Drop dynamics after impact on a solid wall: Theory and simulations, Phys. Fluids 22, 062101 (2010).

[28] S. von Kann, J. H. Snoeijer, and D. van der Meer, Velocity oscillations and stop-go cycles: The trajectory of an object settling in a cornstarch suspension, Phys. Rev. E 87, 042301 (2013).

[29] F. Boyer, O. Pouliquen, and E. Guazzelli, Dense suspensions in rotating-rod flows: Normal stresses and particle migration, J. Fluid Mech. 686, 5 (2011).

[30] G. Ovarlez, F. Bertrand, and S. Rodts, Local determination of the constitutive law of a dense suspension of noncolloidal particles through magnetic resonance imaging, J. Rheol. 50, 259 (2006).

[31] V. Bertola and M. D. Haw, Impact of concentrated colloidal suspension drops on solid surfaces, Powder Technol. 270, 412 (2015). 